\title{
Tight Robust interval observers: an LP approach
}

\author{
M. Ait Rami, C. H. Cheng and C. de Prada
}

\begin{abstract}
This paper provides a solution based on Linear Programming to the problem of designing observers that ensures guaranteed bounds on the estimated states. Firstly, considering linear systems without uncertainties, we provide a complete solution for the existence of interval observers having minimal $l_{1}$-norm of the interval error. Secondly, new type of observers involving dilatation functions, are introduced in order to deal with the robust estimation of systems that are possibly nonlinear and subject to uncertainties. A new methodology is provided for the design and characterization of tight robust interval observers. All the proposed conditions are expressed in term of Linear programming.
\end{abstract}

Key Words: Luenberger observers, dilatation observers, interval observers, linear programming.

\section{INTRODUCTION}

Since the seminal works [22], [23], [20], the estimation problem has been thoroughly studied in the literature. Tremendous efforts have been deployed for the design of estimators (observers), involving many generalizations and approaches in order to deal with nonlinearity and/or robustness in the presence of uncertaities. For instance, without been exhaustive, the design of estimators or observers is based on different geometrical regions involving an appropriate machinery, as a box [18], an ellipsoid [8], [9], [10], [15], [14], a parallelotope [11], a set-valued [26], a zonotope [25], [6], [5], the nonnegative $\mathbf{R}^{n}$ orthant [2], [3], [4], [12], [17], [27], [7].

In this paper, we consider the so-called bounding observation problem or interval observers reconstruction problem, which consists of designing observers that provide guaranteed bounds on the estimated states [5], [6], [15], [19], [18]. Our design of interval observers lies on the theory of positive systems (see [16], [21], [24], [13], [3] for general references). In fact, enforcing the positivity of the error estimation will necessary lead to a guaranteed bounds on the estimated states, once we start with a priori bounds on the unknown initial condition of the observed system. Here, we provide a simple and efficient way of designing observers that ensures guaranteed bounds on the estimated states (Interval Observers). The proposed methodology is based on the Linear Programming (LP) approach initiated in [1], [2], [3], [4]. However, we provide a new reformulation of the LP conditions.

In the first place, we consider linear systems without uncertainties, and look for an easy computable solution for

M. Ait Rami and C. de Prada are with the Departamento de Ingenieria de Sistemas y Automatica, University of Valladolid, 47005 Valladolid, Spain, e-mail:prada@autom.uva.es, aitrami@autom.uva.es

C. H. Cheng is with the Dept. of Systems Engineering and Engineering Management, Chinese University of Hong Kong, Hong Kong, email:chcheng@se.cuhk.edu.hk the existence of interval observers. The obtained conditions are checkable necessary and sufficient conditions in terms of LP. Also, the minimization of the $l_{1}$-norm of the interval error, will be stated and solved as a linear program. In second place, a new type of robust observers involving dilatation functions: Dilatation Observers, are introduced in order to deal with the robust estimation for systems that are possibly nonlinear and subject to uncertainties. In addition, new methodology is provided for the design and characterization of a special type of dilatation observers with guaranteed tight estimated error.

The remainder of the paper is organized as follows. In section 2, the objective of the paper is stated and some preliminary results are given. Section 3 treats the existence and convergence of interval observers without introducing uncertainties in the model. Section 4 introduces the concept of dilatation observers in order to deal with the interval estimation problem for systems involving nonlinearities and/or subject to uncertainties. Section 5 studies a numerical example. Finally, section 6 gives some concluding remarks.

Basic Notation: $\mathbf{R}_{+}^{n}$ denotes the non-negative orthant of the $n$-dimensional real space $\mathbf{R}^{n} . M^{T}$ denotes the transpose of the real matrix $M$. For a real matrix $M, M>0$ means that its components are positive: $M_{i j}>0$, and $M \geq 0$ means that its components are nonnegative: $M_{i j} \geq 0$.

\section{Preliminaries}

This section exposes the objective under investigation and provides some definitions and preliminary results that are used throughout the paper.

\section{A. Problem formulation}

The paper is devoted to the estimation of the states of systems which can be expressed as:

$$
\begin{aligned}
& \dot{x}(t)=A(\delta) x(t)+\xi(x, \delta, t), \\
& y(t)=C x(t),
\end{aligned}
$$

where the matrix $A$ and the nonlinear term $\xi$ possibly depend on unknown parameter $\delta$.

First, let us start with the following general definition.

Definition 2.1: An interval observer for system (1) is a pair of lower and upper estimator functions $\left\{\hat{x}^{l}(\cdot), \hat{x}^{u}(\cdot)\right\}$ of the real state function $x(\cdot)$, that is

$$
\hat{x}^{l}(t) \leq x(t) \leq \hat{x}^{u}(t), \forall t \geq 0 .
$$

In the sequel, we will also investigate the convergence of interval observers and make use of the following definitions.

Definition 2.2: An interval observer for system (1) is said to be convergent if the limit $l=\lim _{t \rightarrow+\infty} \hat{x}^{l}(t)-\hat{x}^{u}(t)$ exists or bounded. 
Definition 2.3: An interval observer for system (1) is said to be asymptotically convergent if $\lim _{t \rightarrow+\infty} \hat{x}^{l}(t)-\hat{x}^{u}(t)=0$.

The objective is to design convergent interval observers for models of the form (1), in the case when the initial conditions are not known but bounded

$$
\bar{x}_{0} \leq x(0) \leq \underline{x}_{0},
$$

and some parameters of the dynamical model (1) are not perfectly known but bounded.

In fact, the main idea behind the proposed interval observers machinery is to reconstruct the error dynamics of the estimators in such way that it can be governed by a differential dynamical equation: $\dot{e}=(A-L C) e+g$ which enforced to be inherently positive by choosing an adequate matrix gain $L$. That is, we have to ensure that if the initial error is nonnegative $e(0) \geq 0$, then error will remain nonnegative $e(t) \geq 0$ all the time. Consequently, by using this positivity concept it will be shown that we can ensure a robust estimation with guaranteed lower and upper bounds on the observed states.

\section{B. Positivity}

Consider the following autonomous linear system:

$$
\dot{x}(t)=M x(t),
$$

where $M$ is a constant matrix and the initial conditions are assumed to be nonnegative $x(0) \in \mathbf{R}_{+}^{n}$.

In order to use a common vocabulary, the following definitions will be used throughout the paper.

Definition 2.4: System (2) is said to be a positive system if the corresponding trajectory is nonnegative: $x(t) \geq 0$ for all $t \geq 0$ and all nonnegative initial conditions $x(0) \geq 0$.

Definition 2.5: A real matrix $M$ is called a Hurwitz matrix if all its eigenvalues have a real strictly negative part.

Definition 2.6: A real matrix $M$ is called a Metzler matrix if all its off-diagonal entries are nonnegative: $M_{i j} \geq 0, i \neq j$.

The following classical result is connected to the positivity of system 2

Lemma 2.1: [23] System (2) is positive if and only if $M$ is a Metzler matrix: $M_{i j} \geq 0, i \neq j$.

The following stability results will play an essential role for the analysis and synthesis of the observers convergence.

Theorem 2.1: [3] If System (2) is positive, then the following statements are equivalent:

- (i) System (2) is asymptotically stable for every initial condition $x(0) \in \mathbf{R}^{n}$ (i.e: $M$ is a Hurwitz matrix).

- (ii) System (2) is asymptotically stable for every nonnegative initial condition $x(0) \in \mathbf{R}_{+}^{n}$.

- (iii) System (2) is asymptotically stable for some initial condition $x(0)$ in the interior of $\mathbf{R}_{+}^{n}$.

- (iv) There exists $\lambda \in \mathbf{R}_{+}^{n}$ such that $M \lambda<0$.

An immediate consequence of Theorem 2.1 is stated as follows.

Theorem 2.2: If System (2) is positive (or equivalently $M$ is Metzler), then the following statements are equivalent:

- (i) System (2) is asymptotically stable for every initial condition $x(0) \in \mathbf{R}^{n}$ (i.e: $M$ is a Hurwitz matrix).
- (ii) There exists $\lambda \in \mathbf{R}_{+}^{n}$ solution to

$$
M \lambda=-\left[\begin{array}{c}
1 \\
\vdots \\
1
\end{array}\right], \lambda>0 .
$$

Proof: One can mimics the proof of Theorem 2.1 given in [3] by choosing $x_{0}^{T}=\left[\begin{array}{lll}1 & \ldots & 1\end{array}\right]$ as initial condition.

\section{EXACT INTERVAL OBSERVERS}

Consider the following system:

$$
\begin{aligned}
\dot{x}(t) & =A x(t)+\xi(y(t), t), \\
y(t) & =C x(t),
\end{aligned}
$$

It is well-known that an observer which converges to the real state possessing a linear error dynamics can be designed in the Luenberger form [22], [23]:

$$
\dot{\hat{x}}=A \hat{x}+\xi(y, t)+L(y-C \hat{x})
$$

where $L$ is the observer gain matrix to be designed. The reconstruction error dynamics is governed by the following linear dynamical equation : $\dot{e}=(A-L C) e$. Consequently, the observer convergence and characteristic properties depend only on the gain matrix $L$ which can be adequately designed in order to obtain an interval observer of system (3).

\section{A. Computation of Interval Observers}

Here, the purpose is to show how to design interval observers by using Linear programming. The following key role result will be used in the sequel.

Proposition 3.1: For any given bound on the initial value of system (3), $\bar{x}_{0} \leq x(0) \leq \underline{x}_{0}$ there exists an asymptotically convergent interval observer $\left\{\hat{x}^{l}(\cdot), \hat{x}^{u}(\cdot)\right\}$ of the form:

$$
\left\{\begin{array}{l}
\dot{\hat{x}}^{l}=A \hat{x}^{l}+\xi(y, t)+L\left(y-\hat{x}^{l}\right), \hat{x}^{l}(0)=\underline{x}(0) \\
\hat{\hat{x}}^{u}=A \hat{x}^{u}+\xi(y, t)+L\left(y-\hat{x}^{u}\right), \hat{x}^{u}(0)=\bar{x}(0),
\end{array}\right.
$$

if and only if $A-L C$ is a Metzler and Hurwitz matrix.

Proof: Sufficiency: We have to show that the upper error $e_{+}=\hat{x}^{u}-x$ and the lower error $e_{-}=x-\hat{x}^{l}$ are positive and converges to zero. Since the upper error satisfies $\dot{e}_{+}=(A-L C) e_{+}$and $A-L C$ is Metzler, it suffices to use Lemma 2.1 to show the nonnegativity of the estimated error. The necessity part and the rest of the proof are straightforward.

Now, for the computation of the interval observer of the form (5) we provide a constructive methodology based on Linear programming.

Theorem 3.1: For any given bound on the initial value of system (3), $\bar{x}_{0} \leq x(0) \leq \underline{x}_{0}$ there exists an asymptotically convergent interval observer $\left\{\hat{x}^{l}(\cdot), \hat{x}^{u}(\cdot)\right\}$ of the form (5), if and only if the following LP problem in the variables $\lambda \in \Re^{n}$ and $Z \in \Re^{r \times n}$ is feasible:

$$
\left\{\begin{array}{l}
A^{T} \lambda-C^{T} Z\left[\begin{array}{c}
1 \\
\vdots \\
1
\end{array}\right]<0, \\
\lambda>0, \\
A^{T} \operatorname{diag}(\lambda)-C^{T} Z+I \geq 0 .
\end{array}\right.
$$


Moreover, a gain matrix $L$ can be computed as follows

$$
L=\operatorname{diag}(\lambda)^{-1} Z^{T},
$$

where the vector $\lambda$ and the matrix $Z$ constitute any feasible solution to the above LP problem.

Proof: It is clear that by using the preceding Theorem 3.1, we have to prove that there exits a matrix gain $L$ such that $A-L C$ is a Metzler and Hurwitz matrix.

Sufficiency: If the LP conditions (6) are satisfied, then the third inequality is equivalent to the positivity of the error. Multiplying it by $\operatorname{diag}(\lambda)^{-1}$ gives $A^{T}-C^{T} L^{T}+$ $\operatorname{diag}(\lambda)^{-1} \geq 0$ or equivalently that $A-L C$ is a Metzler matrix. In addition, the first and second inequalities ensure asymptotic stability of the error. To see that we substitute $Z=L^{T} \operatorname{diag}(\lambda)$ into $A^{T} \lambda-C^{T} Z\left[\begin{array}{c}1 \\ \vdots \\ 1\end{array}\right]<0$ and obtain $\left(A^{T}-C^{T} L^{T}\right) \lambda<0$, which means by using Theorem 2.1 that $(A-L C)^{T}$ is a Hurwitz matrix, or equivalently, that $A-L C$ is a Hurwitz matrix.

Necessity: Let $L$ be the gain of the interval observer (5). Then, by using lemma 2.1 the transpose matrix $(A-L C)^{T}$ must be a Hurwitz and Metzler matrix. Now, using Theorem 2.1 there exists $\lambda>0$ such that $(A-L C)^{T} \lambda<0$. . Thus, by making the change of variables $Z=L^{T} \operatorname{diag}(\lambda)$ the first two inequalities in the LP conditions (6) are obtained. The Last inequality by multiplying $(A-L C)^{T}$ by $\operatorname{diag}(\lambda)$ and obtain the associated Meztler matrix $A^{T} \operatorname{diag}(\lambda)-C^{T} Z$. Then, by using the fact that the vector $\lambda$ can be chosen with sufficiently small components (using homogeneity of the stability condition) we obtain $A^{T} \operatorname{diag}(\lambda)-C^{T} Z+I \geq 0$ and the proof is complete.

\section{B. Optimal Interval Observers}

Our aim is to design interval observers with minimal interval error. This can be done by minimizing the $l_{1}$-norm of the interval error:

$$
\|e\|_{1}=\int_{0}^{+\infty}\left[\begin{array}{lll}
1 & \ldots & 1
\end{array}\right] e(s) d s .
$$

A preliminary explanation on the know-how handling the optimization of the $l_{1}$-norm $\|e\|_{1}$ should be given. Note that by integrating the equation $\dot{e}=(A-L C) e$ we have that the integral of the interval error satisfies

$$
(A-L C) \int_{0}^{+\infty} e(s) d s=\underline{x}_{0}-\bar{x}_{0},
$$

Thus, the the integral of the interval error is given by the formula

$$
\int_{0}^{+\infty} e(s) d s=(A-L C)^{-1}\left(\underline{x}_{0}-\bar{x}_{0}\right) .
$$

In conclusion, the $l_{1}$-norm of the interval error can be expressed as

$$
\begin{aligned}
\|e\|_{1} & =\left[\begin{array}{ccc}
1 & \ldots & 1
\end{array}\right](A-L C)^{-1}\left(\underline{x}_{0}-\bar{x}_{0}\right) \\
& =\left(\underline{x}_{0}-\bar{x}_{0}\right)^{T}(A-L C)^{-T}\left[\begin{array}{lll}
1 & \ldots & 1
\end{array}\right]^{T}
\end{aligned}
$$

We stress out that right now this expression is nonlinear in the gain $L$ of the interval observer.

By using the vector $\lambda$ solution to

$$
(A-L C)^{T} \lambda=-\left[\begin{array}{c}
1 \\
\vdots \\
1
\end{array}\right], \lambda>0,
$$

which always exists by the fact that $A-L C$ is Metzler and Hurwitz (see for this claim Theorem 2.2). Then, $\lambda$ is simply expressed as

$$
\lambda=-(A-L C)^{-T}\left[\begin{array}{c}
1 \\
\vdots \\
1
\end{array}\right]
$$

Now, by using the formula (7) we like to reformulate the expression of the $l_{1}$-norm $\|e\|_{1}$ as

$$
\|e\|_{1}=\left(\underline{x}_{0}-\bar{x}_{0}\right)^{T} \lambda \text {, }
$$

for which $\lambda$ satisfies the stability condition (8). Thus, we arrived at the ultimate point and a linear objective to be minimized is obtained.

Based on the preceding consideration, we are in place to minimize the $l_{1}$-norm $\|e\|_{1}$ and then, here is a way to do it.

Theorem 3.2: For any given bound on the initial value of system (3), $\bar{x}_{0} \leq x(0) \leq \underline{x}_{0}$ the tightest asymptotically convergent interval observer $\left\{\hat{x}^{l}(\cdot), \hat{x}^{u}(\cdot)\right\}$ of the form:

$$
\begin{gathered}
\dot{\hat{x}}^{l}=A \hat{x}^{l}+\xi(y, t)+L\left(y-\hat{x}^{l}\right), \hat{x}^{l}(0)=\underline{x}_{0} \\
\dot{\hat{x}}^{u}=A \hat{x}^{u}+\xi(y, t)+L\left(y-\hat{x}^{u}\right), \hat{x}^{u}(0)=\bar{x}_{0},
\end{gathered}
$$

can be computed from the minimal solution to

$$
\left(\mathcal{P}_{1}\right):\left\{\begin{array}{l}
\min _{L \in \Re^{n \times r}}\left\|\hat{x}^{u}-\hat{x}^{l}\right\|_{1} \\
\text { subject to: } \\
A-L C \text { is Metzler and Hurwitz matrix. }
\end{array}\right.
$$

Or equivalently, the associated optimal gain $L$ can be calculated from the optimal solution to the following optimization LP problem in the variables $\beta \in \Re, \lambda \in \Re^{n}$ and $Z \in \Re^{r \times n}$ :

$$
\left(\mathcal{P}_{2}\right):\left\{\begin{array}{l}
\min \left(\bar{x}_{0}-\underline{x}_{0}\right)^{T} \lambda \\
\text { subject to: } \\
A^{T} \lambda-C^{T} Z\left[\begin{array}{c}
1 \\
\vdots \\
1
\end{array}\right]=-\left[\begin{array}{c}
1 \\
\vdots \\
1
\end{array}\right], \\
\lambda>0, \\
A^{T} \operatorname{diag}(\lambda)-C^{T} Z+\beta I \geq 0 .
\end{array}\right.
$$

Moreover, any solution to $\left(\mathcal{P}_{1}\right)$ corresponds to a solution to $\left(\mathcal{P}_{2}\right)$ and viceversa the optimal gain matrix $L$ of $\left(\mathcal{P}_{1}\right)$ can be computed as follows

$$
L=\operatorname{diag}(\lambda)^{-1} Z^{T}
$$

where the vector $\lambda$ and the matrix $Z$ are any optimal solution to $\left(\mathcal{P}_{2}\right)$.

Proof: Here is a sketch of the proof. Take into account the previous explanations by using the formula (9) then 
combine it with the result of Theorem 2.2. Thus, it suffices to follow a similar proof as for Theorem 3.1. But in contrast, it is emphasized that the first inequality in the proposed LP conditions (11), is not homogeneous in the variable $\lambda$, so that we need to add an extra slack scalar variable $\beta$ to ensure that the matrix $A-L C$ is Metzler.

\section{ROBUST INTERVAL OBSERVERS}

Consider the following uncertain nonlinear system in the form:

$$
\begin{aligned}
\dot{x}(t) & =A(\delta) x(t)+\xi(x, \delta, t), \\
y(t) & =C x(t),
\end{aligned}
$$

where the matrix $A(\delta) \in \mathbf{R}^{n \times n}$ is assumed to be unknown and depending on an uncertain parameter $\delta$ and $\xi(x, \delta, t)$ is the nonlinear part.

Remark 4.1: We stress out that the proposed results also apply to any kind of nonlinear systems

$$
\dot{x}=f(x, \delta, t)
$$

which can be split into the form of system (12).

In the next, we define our dilatation concept that will be utilized for the design of dilatation observers.

\section{A. Dilatation Observers}

Definition 4.1: A function $\psi(\cdot): \mathbf{R}^{n} \rightarrow \mathbf{R}^{n}$ is called a positive dilatation if it satisfies

$$
\psi(x) \geq x, \quad \psi(x) \geq 0 \quad \forall x \in \mathbf{R}^{n} .
$$

A function $\phi(\cdot): \mathbf{R}^{n} \rightarrow \mathbf{R}^{n}$ is called a negative dilatation if it satisfies

$$
x \geq \phi(x), \quad \phi(x) \leq 0 \quad \forall x \in \mathbf{R}^{n} .
$$

Based on theses definitions, we introduce and define our dilatation observers which are given by the following two nonlinear dynamical systems

$$
\left\{\begin{array}{l}
\dot{\hat{x}}^{l}=\bar{A} \hat{x}^{l}+L\left(y-C \hat{x}^{l}\right)-(\bar{A}-\underline{A}) \psi\left(\hat{x}^{l}\right)+\xi \\
\dot{\hat{x}}_{u}=\bar{A} \hat{x}^{u}+L\left(y-C \hat{x}^{u}\right)-(\bar{A}-\underline{A}) \phi\left(\hat{x}^{u}\right)+\bar{\xi},
\end{array}\right.
$$

where the functions $\psi$ and $\phi$ are respectively positive and negative dilatations (with regard to Definition 4.1). The functions $\underline{\xi}$ and $\bar{\xi}$ are obtained by adequately substituting $\xi$ by any known upper and lower bounds on it. The matrices $\underline{A}, \bar{A}$ are assumed to be constant and such that

$$
\underline{A} \leq A(\delta) \leq \bar{A}
$$

Theorem 4.1: For any given bounds on the initial value of system (12), $\underline{x}_{0} \leq x_{0} \leq \bar{x}_{0}$ and any given functions $\psi$ and $\phi$ which are respectively positive and negative dilatations, then there exists a robust interval observer of the form:

$$
\left\{\begin{array}{l}
\dot{\hat{x}}^{l}=\bar{A} \hat{x}^{l}+L\left(y-C \hat{x}^{l}\right)-(\bar{A}-\underline{A}) \psi\left(\hat{x}^{l}\right)+\underline{\xi} \\
\dot{\hat{x}}_{u}=\bar{A} \hat{x}^{u}+L\left(y-C \hat{x}^{u}\right)-(\bar{A}-\underline{A}) \phi\left(\hat{x}^{u}\right)+\bar{\xi} \\
\hat{x}^{l}(0)=\underline{x}_{0}, \quad \hat{x}^{u}(0)=\bar{x}_{0},
\end{array}\right.
$$

if the matrix $\underline{A}-L C$ is Metzler.

Proof: To simplify the notation, we define $e_{-}=x-\hat{x}^{l}$, $e_{+}=\hat{x}^{u}-x, \xi_{-}=\xi-\underline{\xi}$ and $\xi_{+}=\bar{\xi}-\xi$. We have to show that if the matrix $\underline{A}-L C$ is Metzler then we have necessary $e_{+} \geq 0$ and $e_{-} \geq 0$ whenever $e_{+}(0) \geq 0$ and $e_{-}(0) \geq 0$.

Since the lower and upper errors $e_{-}$and $e_{+}$satisfy

$$
\begin{gathered}
\dot{e}_{-}=(A-L C) e_{-}+(A-\bar{A}) \hat{x}^{l}+(\bar{A}-\underline{A}) \psi\left(\hat{x}^{l}\right)+\xi_{-}, \\
\dot{e}_{+}=(A-L C) e+(\bar{A}-A) \hat{x}^{u}-(\bar{A}-\underline{A}) \phi\left(\hat{x}^{u}\right)+\xi_{+},
\end{gathered}
$$

If $\underline{A}-L C$ is Metzler then $A-L C$ is Metzler for any $A$ in the interval

$$
\underline{A} \leq A(\delta) \leq \bar{A}
$$

Therefore, since $\xi_{-} \geq 0, \xi_{+} \geq 0$, then it suffices to show the nonnegativity of the following terms

$$
\begin{gathered}
v_{1}=(A-\bar{A}) \hat{x}^{l}+(\bar{A}-\underline{A}) \psi\left(\hat{x}^{l}\right) \geq 0, \\
v_{2}=(\bar{A}-A) \hat{x}^{u}-(\bar{A}-\underline{A}) \phi\left(\hat{x}^{u}\right) \geq 0 .
\end{gathered}
$$

Now, since $\psi\left(\hat{x}^{l}\right) \geq \hat{x}^{l}$ and $A-\bar{A} \leq 0$, this implies $(A-\bar{A}) \hat{x}^{l} \geq(A-\bar{A}) \psi\left(\hat{x}^{l}\right)$. So by using the positivity of the dilatation function $\psi(\cdot) \geq 0$ and the fact that $A-\underline{A} \geq 0$, we can see that

$$
\begin{gathered}
v_{1} \geq(A-\bar{A}) \psi\left(\hat{x}^{l}\right)+(\bar{A}-\underline{A}) \psi\left(\hat{x}^{l}\right), \\
v_{1} \geq(A-\underline{A}) \psi\left(\hat{x}^{l}\right) \geq 0 .
\end{gathered}
$$

In the same manner, since $\hat{x}^{u} \leq \phi\left(\hat{x}^{u}\right) \geq$ and $\bar{A}-A \geq 0$, we have $(\bar{A}-A) \hat{x}^{u} \geq(\bar{A}-A) \phi\left(\hat{x}^{l}\right)$. So regarding to the negativity of the dilatation function $\phi(\cdot) \leq 0$ and the fact that $\underline{A}-A \geq 0$, we obtain

$$
\begin{gathered}
v_{2} \geq(\bar{A}-A) \phi\left(\hat{x}^{u}\right)-(\bar{A}-\underline{A}) \phi\left(\hat{x}^{u}\right), \\
v_{2} \geq(\underline{A}-A) \phi\left(\hat{x}^{l}\right) \geq 0,
\end{gathered}
$$

and the proof is complete.

The previous result will be applied to a very interesting interval observers involving a special kind of dilatation functions

$$
\begin{aligned}
& d^{+}(x)=0.5(x+|x|), \\
& d^{-}(x)=0.5(x-|x|),
\end{aligned}
$$

where $|x|$ denotes the vector formed by the absolute values of the components of the vector $x \in \Re^{n}$.

\section{B. Tight Robust Interval Observers}

In order to derive the main result of this section, we will use the following key role Lemma.

Lemma 4.1: Consider the following differential system

$$
\dot{v}=M v+g(v, t) \text {, where } M \text { is a Metzler matrix. }
$$

Then, if $g(v, t) \leq \tilde{g}$ we have

$$
v(t) \leq \tilde{v}(t), \quad \forall t \geq 0,
$$

where $\tilde{v}(\cdot)$ is the solution of the differential equation

$$
\dot{\tilde{v}}=M \tilde{v}+\tilde{g}, \quad \tilde{v}(0)=v(0)=v_{0} .
$$

Proof: Omitted because of the lack of space.

Now, we are in place to state the main result of this section.

Theorem 4.2: Assume that the trajectory of system (12) is bounded.

$$
|x(t)| \leq m, \quad \forall t \geq 0
$$


Then, for any given bound on the initial value of system (12), $\underline{x}_{0} \leq x_{0} \leq \bar{x}_{0}$, the following special robust interval observer of the form:

$$
\left\{\begin{array}{l}
\dot{\hat{x}}^{l}=\bar{A} \hat{x}^{l}+L\left(y-\hat{x}^{l}\right)-(\bar{A}-\underline{A}) d^{+}\left(\hat{x}^{u}\right)+\xi \\
\hat{\hat{x}}_{u}=\bar{A} \hat{x}^{u}+L\left(y-\hat{x}^{u}\right)-(\bar{A}-\underline{A}) d^{-}\left(\hat{x}^{l}\right)+\bar{\xi}, \\
\text { where } d^{+}, d^{-} \text {are given by }(14),
\end{array}\right.
$$

is a convergent robust interval observer if the matrix $\underline{A}-L C$ is Metzler and the matrix $\bar{A}-L C$ is Hurwitz. Moreover, we have that

$$
\limsup _{t \rightarrow+\infty} \hat{x}^{u}(t)-\hat{x}^{l}(t) \leq 2(\bar{A}-L C)^{-1}[(\bar{A}-\underline{A}) m+\bar{\xi}-\underline{\xi}] .
$$

Proof: Let $e=\hat{x}^{u}-\hat{x}^{l}$ be the interval error, which satisfies

$$
\dot{e}=(\bar{A}-L C) e+(\bar{A}-\underline{A})\left(d^{+}\left(\hat{x}^{l}\right)-d^{-}\left(\hat{x}^{u}\right)\right)+\bar{\xi}-\underline{\xi} .
$$

Regarding to the fact that $d^{+}(\cdot), d^{-}(\cdot)$ are dilatation functions then by using Theorem 4.1, we have

$$
\hat{x}^{l}(t) \leq x(t) \leq \hat{x}^{u}(t), \quad \forall t \geq 0 .
$$

By using the assumption $|x(t)| \leq m$ and the property that $d^{+}(\cdot)$ involves only the nonnegative components of $\hat{x}^{u}$ and $d^{-}(\cdot)$ involves only the negative components of $\hat{x}^{l}$, we necessarily have

$$
d^{+}\left(\hat{x}^{l}(t)\right) \leq m \text { and }-d^{-}\left(\hat{x}^{u}(t)\right) \leq m, \quad \forall t \geq 0 .
$$

Now, consider the linear differential equation

$$
\dot{v}=(\bar{A}-L C) v+2(\bar{A}-\underline{A}) m+\bar{\xi}-\underline{\xi}, v(0)=e(0),
$$

and note that $\bar{A}-L C$ is Metzler since $\underline{A}-L C$ is Metzler. Then, by using the comparison Lemma 4.1 we obtain

$$
e(t) \leq v(t), \quad \forall t \geq 0 .
$$

Now, since $\bar{A}-L C$ is Hurwitz, $v(t)$ converge to the only equilibrium steady state $\bar{v}$ solution to

$$
(\bar{A}-L C) \bar{v}+2(\bar{A}-\underline{A}) m+\bar{\xi}-\underline{\xi}=0,
$$

or equivalently

$$
\bar{v}=2(\bar{A}-L C)^{-1}[(\bar{A}-\underline{A}) m+\bar{\xi}-\underline{\xi}] .
$$

Since we showed that $e(t) \leq v(t), \forall t \geq 0$, consequently, we obtain

$\limsup _{t \rightarrow+\infty} \hat{x}^{u}(t)-\hat{x}^{l}(t) \leq 2(\bar{A}-L C)^{-1}[(\bar{A}-\underline{A}) m+\bar{\xi}-\underline{\xi}]$.

The previous result can be improved by minimizing the derived bound on the interval observer:

$$
2(\bar{A}-L C)^{-1}(\bar{A}-\underline{A}) m+\bar{\xi}-\underline{\xi} .
$$

This is possible by using the following result.

Theorem 4.3: A sharpest bound on the interval observer $\left\{\hat{x}^{l}(\cdot), \hat{x}^{u}(\cdot)\right\}$ of the form (15) can be computed from the optimal solution to the following LP problem in the variables $\beta \in \Re, \lambda \in \Re^{n}$ and $Z \in \Re^{r \times n}$ is achievable:

$$
\left(\mathcal{P}_{3}\right):\left\{\begin{array}{l}
\min [(\bar{A}-\underline{A}) m+\bar{\xi}-\underline{\xi}]^{T} \lambda \\
\text { subject to: } \\
\bar{A}^{T} \lambda-C^{T} Z\left[\begin{array}{c}
1 \\
\vdots \\
1
\end{array}\right]=-\left[\begin{array}{c}
1 \\
\vdots \\
1
\end{array}\right], \\
\lambda>0, \\
\underline{A}^{T} \operatorname{diag}(\lambda)-C^{T} Z+\beta I \geq 0 .
\end{array}\right.
$$

Moreover, the gain matrix $L$ of the interval observer (15) can be computed as follows

$$
L=\operatorname{diag}(\lambda)^{-1} Z^{T},
$$

where the vector $\lambda$ and the matrix $Z$ are any feasible solution to the above LP problem.

\section{Numerical Example}

We consider an uncertain nonlinear system subject to a parametric perturbation as follows:

$$
\begin{aligned}
\dot{x}(t) & =A(\delta) x(t)+\delta \sin \left[x_{1}(t)^{2}\right]\left[\begin{array}{l}
1 \\
1 \\
1
\end{array}\right], \\
y(t) & =\left[\begin{array}{lll}
1 & 1 & 0
\end{array}\right] x(t),
\end{aligned}
$$

where the matrix $A(\delta)$ is given by

$$
A(\delta)=\left[\begin{array}{ccc}
-2.5 & 0.2 & 1 \\
0.1 & -0.5 & 1 \\
0 & 0.3 & -0.8-0.01 \delta
\end{array}\right],
$$

and the uncertain parameter satisfies $0 \leq \delta \leq 1$.

We have to find a robust interval observer of the form (15) which provide guaranteed upper and lower bounds of all the plants between the two extreme plants $(\delta=0$ and $\delta=1)$.

Note that $A(1) \leq A(\delta) \leq A(0)$ and the components of the nonlinear part of system (17) range between -1 and 1 . Thus, a simple choice of a priori bounds on the dynamics of system (17) is as follows: $\underline{A}=A(1), \bar{A}=A(0)$ and

$$
\begin{gathered}
\underline{\xi}=\left[\begin{array}{l}
-1 \\
-1 \\
-1
\end{array}\right], \bar{\xi}=\left[\begin{array}{l}
1 \\
1 \\
1
\end{array}\right], \\
\hat{x}^{l}(0)=\left[\begin{array}{c}
-1.5 \\
1.5 \\
0.5
\end{array}\right], \quad \hat{x}^{u}(0)=\left[\begin{array}{c}
-0.5 \\
2.5 \\
1.5
\end{array}\right] .
\end{gathered}
$$

Then, by applying Theorem 4.3 we have obtained the following gain matrix:

$$
L=\left[\begin{array}{l}
0.2 \\
0.1 \\
0.0
\end{array}\right]
$$

Hence, with this gain, the numerical simulation was carried out by using ODE solver: ODE23 in Matlab. The numerical results for the state evolution of an arbitrary plant corresponding to $\delta=0.5$ and an initial condition $x_{0}=\left[\begin{array}{lll}-1 & 2 & 1\end{array}\right]^{T}$, (which ranges between $\hat{x}^{l}(0)$ and $\left.\hat{x}^{u}(0)\right)$ are depicted in Figure 1, Figure 2 and Figure 3. 


\section{CONCLusions}

In this paper, interval observers reconstruction has been studied and developed. New methodology is proposed for designing observers that ensures guaranteed bounds on the estimated states. We have provided a simple and complete solution to the existence problem of interval observers having fast convergence and minimal $l_{1}$-norm of the interval error for systems without uncertainties. For this case, all the proposed condition are necessarily and sufficient checkable condition in terms of Linear programming. Also, new type of observers involving dilatation functions, has been introduced. It has been shown that by using dilatation observer we can deal with the robust estimation problem for systems that are possibly nonlinear and subject to uncertainties.

\section{REFERENCES}

[1] M. Ait Rami and F. Tadeo. Controller Synthesis for Linear Systems to Impose Positiveness in Closed-Loop States. Proceedings of the IFAC World Congress, Prague, 2005.

[2] M. Ait Rami and F. Tadeo. Discrete positive observers for linear systems. Proceedings of the IEEE Conference on Decision and Control San Diego, 2006.

[3] M. Ait Rami and F. Tadeo. Controller synthesis for positive linear systems with bounded controls. IEEE Transactions on Circuits and Systems II, 54(2), pp. 151-155, 2007.

[4] M. Ait Rami, U. Helmke and F. Tadeo. Positive observation problem for linear time-lag positive systems controls. Proceedings of the IFAC SSSC'07, Brazil, 2007.

[5] T. Alamo, J.M. Bravo, E.F. Camacho. Guaranteed state estimation by zonotopes. Automatica, 41(6): 1035-1043, 2005.

[6] C. Combastel. A state bounding observer based on zonotopes. Proceedings of the of European Control Conference, Cambridge, 2003.

[7] J. Back and A. Astolfi. Positive linear observer design via positive realization. ACC conf., 2007

[8] G. Calafiore. A set-valued non-linear filter for robust localization, ECC, Portugal (2001).

[9] G. Calafiore. Set Simulations for Quadratic Systems. IEEE Transactions on Automatic Control, vol. 48, n. 5, pp. 800-805, 2003

[10] G. Calafiore, L. El Ghaoui. Ellipsoidal Bounds for Uncertain Linear Equations and Dynamical Systems. Automatica, vol. 40, n. 5, pp. 773-787, 2004.

[11] L. Chisci, A. Garulli and G. Zappa. Recursive state bounding by parallelotopes. Automatica 32: 10491055.

[12] N. Dautrebande and G. Bastin. Positive linear observers for positive linear systems. Proceedings of the ECC 99. Karlsruhe, Germany, 1999.

[13] P. de Leenheer and D. Aeyels. Stabilization of positive linear systems. Systems and Control Letters, 44: 861-868, 2001.

[14] C. Durieu, L. Loron, E. Sedda, I. Zein, Fault detection of an induction motor by set-membership filtering and Kalman filtering. Proceedings of the ECC 99. Karlsruhe, Germany, 1999.

[15] C. Durieu, E. Walter and B. Polyak. Multi-Input Multi-Output Ellipsoidal State Bounding. Journal of Optimization Theory and Applications 111(2): 273-303, 2001.

[16] L. Farina and S. Rinaldi. Positive Linear Systems: Theory and Applications. Wiley, New York, 2000.

[17] H.M. Hardin and J.H. van Schuppen. Observers for linear positive systems. Linear Algebra and its Applications, 2007.

[18] J.L. Gouz, A. Rapaport and M.Z. Hadj-Sadok. Interval observers for uncertain biological systems, Ecological Modelling 133: 4556, 2000.

[19] L. Jaulin, M. Kieffer, O. Didrit, E. Walter. Applied Interval Analysis with Examples in Parameter and State Estimation, Robust Control and Robotics. Springer, 2001.

[20] R. Kalman. A new approach to linear filtering and prediction problems. J. of Basic Engineering, 82D: 35-45, 1960.

[21] T. Kaczorek. Positive $1 D$ and $2 D$ Systems. Springer Verlag, UK, 2001.
[22] D. G. Luenberger. Observing the state of a linear system. IEEE Trans. on Military Electronics, 8: 74-80, 1964.

[23] D. G. Luenberger. An introduction to observers. IEEE Trans. Aut. Control, 16: 596-602, 1966.

[24] D. G. Luenberger. Introduction to Dynamic Systems. Wiley, New York 1979.

[25] V. Puig , P. Cuguer, J. Quevedo. Worst-case estimation and simulation of uncertain discrete-time systems using zonotopes. ECC, Portugal, 2001.

[26] J. S. Shamma. Approximate set-value observer for nonlinear systems, IEEE Transactions on Automatic Control, 42: 1997.

[27] J.M. van den Hof. Positive linear observers for linear compartmental systems. SIAM Journal on Control and Optimization, 36(2): 590-608, 1998.

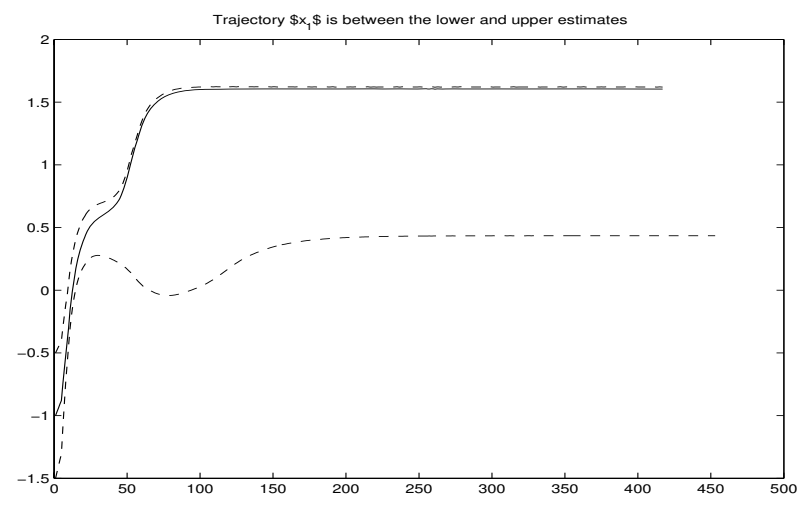

Fig. 1. Evolution of the real state $x_{1}(t)$ (solid line).

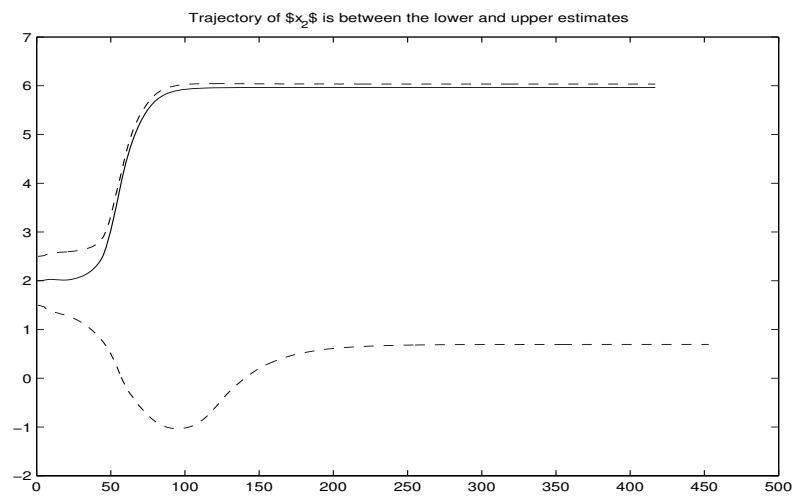

Fig. 2. Evolution of the real state $x_{2}(t)$ (solid line).

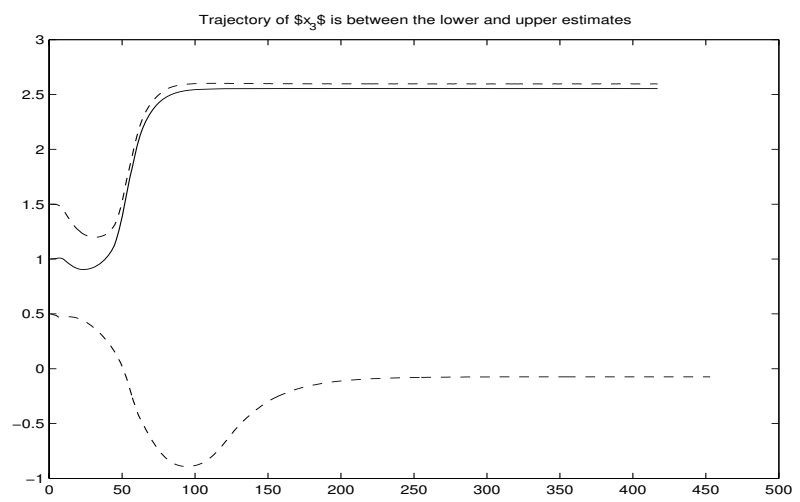

Fig. 3. Evolution of the real state $x_{3}(t)$ (solid line). 